\title{
Naga, ville royale
}

Naga, a royal city

\section{Christian Perzlmeier et Arnulf Schlüter}

Traducteur : Sonia Focke et Anne-Hélène Perrot

\section{OpenEdition} Journals

Édition électronique

URL : http://journals.openedition.org/cel/383

DOI : $10.4000 /$ cel.383

ISSN : 2262-208X

Éditeur

École du Louvre

Référence électronique

Christian Perzlmeier et Arnulf Schlüter, « Naga, ville royale », Les Cahiers de l'École du Louvre [En ligne], 9 | 2016, mis en ligne le 15 décembre 2016, consulté le 17 septembre 2019. URL : http:// journals.openedition.org/cel/383; DOI : 10.4000/cel.383

Ce document a été généré automatiquement le 17 septembre 2019.

\section{(9) $\odot \Theta \Theta$}

Les Cahiers de l'École du Louvre sont mis à disposition selon les termes de la licence Creative Commons Attribution - Pas d'Utilisation Commerciale - Pas de Modification 4.0 International. 


\title{
Naga, ville royale
}

\author{
Naga, a royal city
}

\section{Christian Perzlmeier et Arnulf Schlüter}

Traduction : Sonia Focke et Anne-Hélène Perrot

1 Située à environ 110 kilomètres au nord de Khartoum, au cœur de la steppe de Butana, dans le Wadi Awatib, la ville de Naga est une mine pour les archéologues. Elle était l'une des villes les plus importantes du royaume de Méroé, mais sa localisation étonnante retirée à 30 kilomètres du Nil quand les autres sites antiques se situent généralement près de celui-ci - fait qu'elle est restée intouchée jusqu'à la fin du Xxe siècle. En effet, Naga a, pour cette raison, probablement été épargnée par les réutilisations postérieures, les vols de blocs, la présence de l'agriculture ou encore les fouilles illégales qui sont le sort des autres sites.

2 Naga - Tolkte ${ }^{1}$ en méroïtique - a probablement été fondée dès le début du IV siècle avant J.-C. pour servir de résidence secondaire au royaume dont Méroé était la capitale. À l'apogée du site de Naga, autour du $\mathrm{I}^{\mathrm{er}}$ siècle après Jésus-Christ, la ville s'étendait sur environ $1,2 \mathrm{~km}$.

Naga est la cité la plus méridionale du Royaume de Méroé. Les temples, tous différents les uns des autres, ont été bâtis à peu près au même moment et montrent dans leur structure, leurs éléments architecturaux et leur décoration en relief une diversité d'influences, égyptiennes, romaines et africaines. Cela reflète l'existence des contacts interculturels du Royaume de Méroé, mais aussi sa capacité à assimiler et à comprendre ces cultures extérieures et à produire quelque chose d'unique.

\section{La découverte de Naga²}

3 Appelée "Aithiopia » par les Grecs, "le pays du soleil brûlant », la Nubie antique est mentionnée par différents auteurs anciens : Hérodote, Diodore de Sicile, Strabon, Dion Cassius et Pline. Elle est également citée dans la Bible comme étant le «pays des Maures », dont la « Reine Candace » a envoyé un ambassadeur à Jérusalem³. Par la suite, 
des écrivains comme Jean d'Éphèse, parmi les plus connus, documenteront la christianisation du Soudan au cours du vi ${ }^{\mathrm{e}}$ siècle.

Ce n'est qu'à partir du début du XVIII ${ }^{e}$ siècle que l'Europe redécouvre le Soudan grâce à l'émergence de récits de voyages qui relatent notamment les difficultés rencontrées par ceux qui osèrent entreprendre ce périple, tels que le médecin français Charles-Jacques Poncet $^{4}$ ou le père franciscain Theodor Krump ${ }^{5}$. Aucun des deux ne parvint cependant jusqu'à Naga ; tout comme le célèbre explorateur de l'Afrique, l'Écossais James Bruce ${ }^{6}$ qui, à la recherche des sources du Nil, traversa la région du Chendi en 1769, sans atteindre la cité royale. Il visita néanmoins le site des pyramides de Bégraouia, l'identifiant le premier comme la cité antique de Méroé.

Bien que les temples et les ruines de Naga aient de tous temps été visibles et accessibles, le site semble n'avoir été visité par des Européens qu'à partir du XIX ${ }^{e}$ siècle. Les premiers sont les Français Louis Maurice Adolphe Linant de Bellefonds et Frédéric Cailliaud en 1822. Linant de Bellefonds, qui en tant qu'ingénieur en chef des travaux publics jouera plus tard un rôle fondamental dans la construction du canal de Suez, a parallèlement mené une vie d'explorateur. En juin 1821, il part pour un voyage de treize mois dans le Soudan qui le mène en février 1822 dans la région de Chendi et de Moussaouarat. Bien qu'averti des fréquentes attaques de Bédouins rebelles, il décide cependant de visiter Naga où il arrive le 28 février 1822. Dans ses journaux ${ }^{7}$, il décrit la région et plus particulièrement le Temple du Lion, dont il fournit plusieurs esquisses des reliefs. Après sa mort au Caire en 1883, ses journaux et dessins resteront près d'un siècle sans être publiés, faisant des notices de Cailliaud $^{8}$, qui visita Naga peu après Bellefonds, la première description publiée du site. Le 14 mars 1822, Linant de Bellefonds rencontre à Chendi le minéralogiste Frédéric Cailliaud qu'il encourage à aller visiter Naga, que celui-ci atteint le 22 mars. En moins de quatre jours, il dresse la première carte topographique du lieu, fait des plans d'ensemble des temples visibles et relève plusieurs scènes - ainsi que plusieurs vues d'ensemble de Naga et de l'architecture de ses temples. De retour en France, il publie le récit Voyage à Méroé et au Fleuve Blanc en un volume constitué de 75 planches et de courtes descriptions, qui sera ensuite suivi de quatre volumes de textes. C'est donc Cailliaud qui, le premier, porte à la connaissance du public cette culture ancienne entre Égypte et Afrique.

Plusieurs dessins de Naga sont également effectués par Lord Percy, premier baron de Prudhoe et duc de Northumberland, et par le Major Orlando Felix, lors de leur séjour en $1829^{9}$. Peu de temps après, ils sont suivi de Guiseppe Ferlini, médecin et aventurier de Bologne qui, après avoir quitté l'armée égyptienne en 1834, cherche des objets précieux au Soudan et n'hésite pas, pour cela, à accepter la destruction de monuments entiers ${ }^{10}$. Son séjour à Naga n'est heureusement que de courte durée. En 1835, Sir J. Gardiner Wilkinson et James Burton visitent Naga, ainsi que le peintre Charles Gleyre. Ce dernier a laissé quelques dessins de Naga ${ }^{11}$. Moins de deux ans plus tard, le 26 avril 1837, le célèbre lieutenant-général, paysagiste, écrivain et voyageur, le Prince Hermann Ludwig Heinrich von Pückler-Muskau arrive à Naga ${ }^{12}$. Il était parti d'Alger pour l'Égypte, à l'époque sous le règne de Méhémet Ali Pacha, et de là avait continué son voyage vers le Soudan. Il décrit - avec de nombreuses imprécisions - les monuments, mais n'est particulièrement ravi ni par le lieu, ni par les circonstances de son séjour, rendues difficiles par la chaleur, la fatigue et le manque de provisions. Il laissera tout de même son nom gravé à l'intérieur de la chapelle d'Hathor. Son compagnon de voyage, un certain Max Koch que Méhémet Ali avait attaché à l'expédition en tant que médecin, 
prend le prince comme exemple et grave également son nom sur le pylône du Temple du Lion.

7 L'apogée de l'exploration archéologique du Soudan au XIX siècle reste probablement l'expédition prussienne de 1842 à 1845 que le roi Frédéric Guillaume IV de Prusse envoie explorer l'Égypte, le Sinaï et le Soudan sous le commandement de Karl Richard Lepsius. Le but premier de cette expédition était la documentation de tous les monuments et constructions rencontrés lors du voyage ainsi que la collecte du

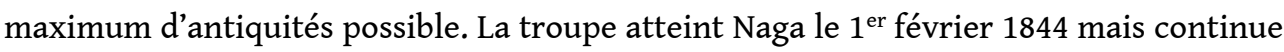
son chemin dès le lendemain vers Moussaouarat. Pendant que Lepsius suit sa route vers Soba et Sennar avec un groupe, plusieurs membres de l'expédition ${ }^{13}$ retournent à Naga pour documenter les monuments visibles, tracer une nouvelle carte topographique et copier les reliefs des Temples d'Amon et du Lion avec l'ensemble de leurs inscriptions ${ }^{14}$.

Quatre ans après Lepsius, P. Trémaux, qui accompagne une expédition de Méhémet Ali au cours supérieur du Nil, arrive à Naga le 22 février 1848 et décrit le lieu dans son récit de voyages ${ }^{15}$. Il semble que les expéditions s'interrompent dans la deuxième moitié du XIX ${ }^{e}$ siècle. Ce n'est qu'après l'insurrection mahdiste (1881-1898) contre l'occupation égyptienne du Soudan, qui fut durement réprimée avec l'aide britannique lors de la bataille sanglante d'Omdourman (1898) que les archéologues reviennent en Nubie. Ce sont dans un premier temps surtout des Anglais et des Américains. Parmi eux, George A. Reisner, qui, même s'il ne visite pas Naga, éveille l'intérêt du monde scientifique pour le Soudan grâce à ses fouilles de sites comme Kerma, le Gébel Barkal et Méroé. Puis E. A. Wallis Budge publie, à la suite de son voyage à Naga en 1903, une description détaillée accompagnée de plans et de dessins du site ${ }^{16}$.

En 1906, l'expédition de James Henry Breasted et Norman de Garis Davis, qui s'étaient surtout consacrés à la documentation des inscriptions, utilise le nouvel outil qu'était la photographie, produisant ainsi une base documentaire qui permet de nos jours, par exemple, de comparer l'état de conservation des monuments sur ces cent dernières années ${ }^{17}$. Francis Llewellyn Griffith, qui travaillait à Méroé pour l'équipe de l'université de Liverpool sous la direction de John Garstang, a lui aussi recopié en 1910 les inscriptions méroïtiques de Naga accessibles à l'époque ${ }^{18}$. Ce n'est que cinquante ans plus tard (en 1957-1958) qu'une entreprise scientifique retourne dans cette région : il s'agit de l'expédition de l'université Humboldt de Berlin sous la direction de Fritz Hintze, qui étudia la grande région du Butana, délimitée par le courant principal du Nil et les deux tributaires issus des montagnes d'Éthiopie, l'Atbara et le Nil Bleu ${ }^{19}$. L'expédition du Butana entreprend pour la première fois des sondages scientifiques à Naga, sondages qui restent modestes. Une tombe de la nécropole qui se situe au sud du Temple d'Amon est néanmoins fouillée à titre d'exemple et les inscriptions du Temple d'Amon et du Temple du Lion sont relevées.

Theodor Kraus se soucie surtout de la Chapelle d'Hathor ${ }^{20}$. Finalement, en 1983, une étude du Temple du Lion en quatre volumes est publiée dans le contexte du Tübinger Atlas des Vorderen Orients, dans lequel Ingrid Gamer-Wallert et Karola Zibelius étudient et publient les reliefs, les inscriptions et l'architecture du temple ${ }^{21}$.

Dietrich Wildung, à cette époque directeur du musée Égyptien de Berlin, reçoit en février 1994 la concession pour développer un ambitieux projet de fouilles à Naga; la première campagne commence dès janvier $1995^{22}$. Les recherches se poursuivent pendant plus de dix-huit ans avant que le projet et les fouilles ${ }^{23}$ soient repris par le musée de Munich en $2013^{24}$. 


\section{La zone urbaine ${ }^{25}$}

La zone de fouilles se situe au pied du Gébel Naga, contrefort méridional d'une chaine de montagnes s'étalant sur plusieurs kilomètres, où se trouve aussi l'ancienne carrière de pierres de la ville de $\mathrm{Naga}^{26}$. Le centre urbain comprend de nombreux temples, palais et bâtiments administratifs, dont trois ont presque intégralement survécu aux ravages du temps : le Temple d'Amon, le Temple du Lion et, devant lui, la Chapelle d'Hathor (fig. 1). D'autres bâtiment sacrés tels que le Temple F, situé au-dessus du temple d'Amon, ou un temple périptère dans la partie sud du site, quoi que moins bien préservés, ont encore une structure clairement reconnaissable. Leur analyse reste un objectif pour les années à venir. Ceci est vrai aussi pour les autres complexes architecturaux, dont le positionnement fait la preuve d'une urbanisation méthodique et qui se présentent sous l'aspect d'un tas de ruines de huit mètres de haut ${ }^{27}$. Dans un large demi-cercle entourant le centre urbain, on trouve des nécropoles comportant des centaines d'inhumations dans des tombes-tumulus grandes et petites. Au milieu du site antique se trouve le puits moderne. Une installation antique comparable n'a pas encore été découverte; pour l'instant, il faut supposer que l'approvisionnement en eau se faisait exclusivement par la collecte d'eau de pluie dans les hafirs ${ }^{28}$ à la lisière sud de la ville.

Fig. 1

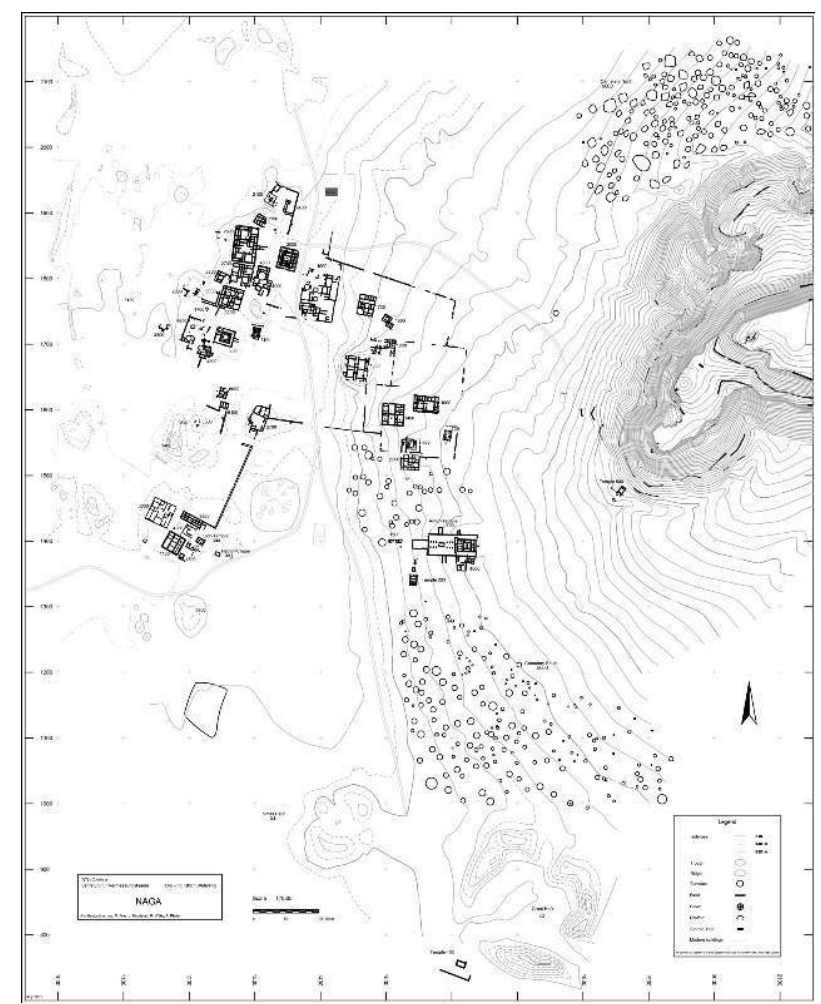

Naga, plan du site

(c) Naga-Project ; Staatlisches Museum Ägyptischer Kunst, München 


\section{Le Temple d'Amon}

12 Le centre urbain se trouve dominé par le temple d'Amon, érigé au $\mathrm{I}^{\mathrm{er}}$ siècle après J.-C. par le roi Natakamani et la reine Amanitore sur une terrasse au-dessous du Gébel Naga. Une large rampe part de l'ouest et monte jusqu'au temple. Avec sa construction axiale qui comprend une allée de béliers (douze statues en tout, fig. 2), un pylône d'entrée imposant ainsi qu'une suite de salles hypostyles, une salle d'offrandes, une salle de la barque et un sanctuaire (fig.3), ce bâtiment correspond en beaucoup de points au concept de base du temple égyptien, mais montre pourtant un grand nombre de détails purement méroïtiques. Dans la zone située devant le temple, à l'ouest de la grande rampe, un autel a été découvert en dehors du temple lui-même ${ }^{29}$, et tout près de là un piédestal pour un trône ${ }^{30}$ portant des représentations d'ennemis subjugués. Un kiosque divise l'allée de béliers en deux moitiés de six statues chacune. Au cours des travaux de restauration, les statues de béliers et leurs bases ont été redressées. Des fragments de statues royales ${ }^{31}$, montées à l'origine entre les pattes avant des béliers, cassées à un moment indéterminé dans le passé, ont été retrouvés lors des fouilles.

Fig. 2

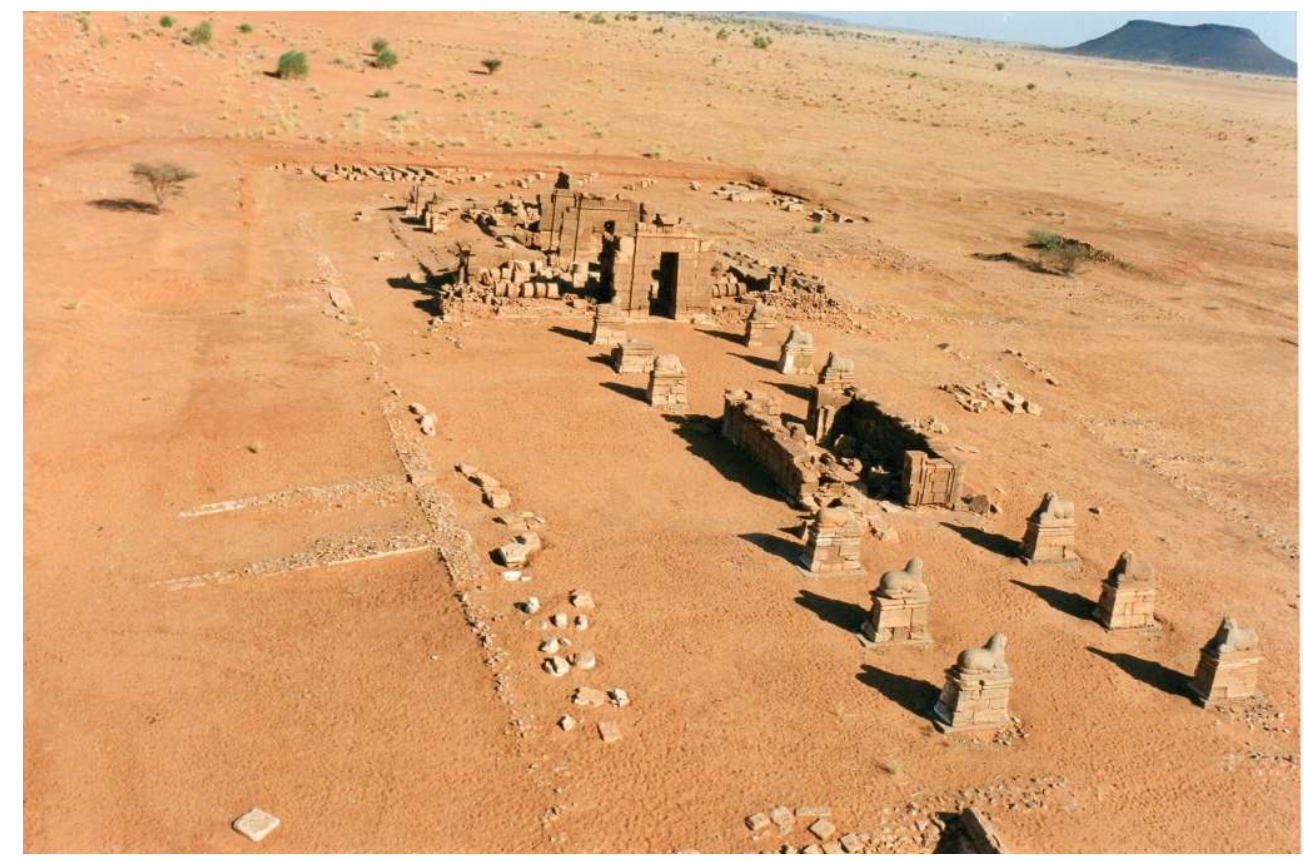

Naga, Temple d'Amon et son allée de béliers

(C) NAgA-PROJECT ; STAATLISCHES MUSEUM ÄgYPTISCHER KUNST, MÜNCHEN 
Fig. 3

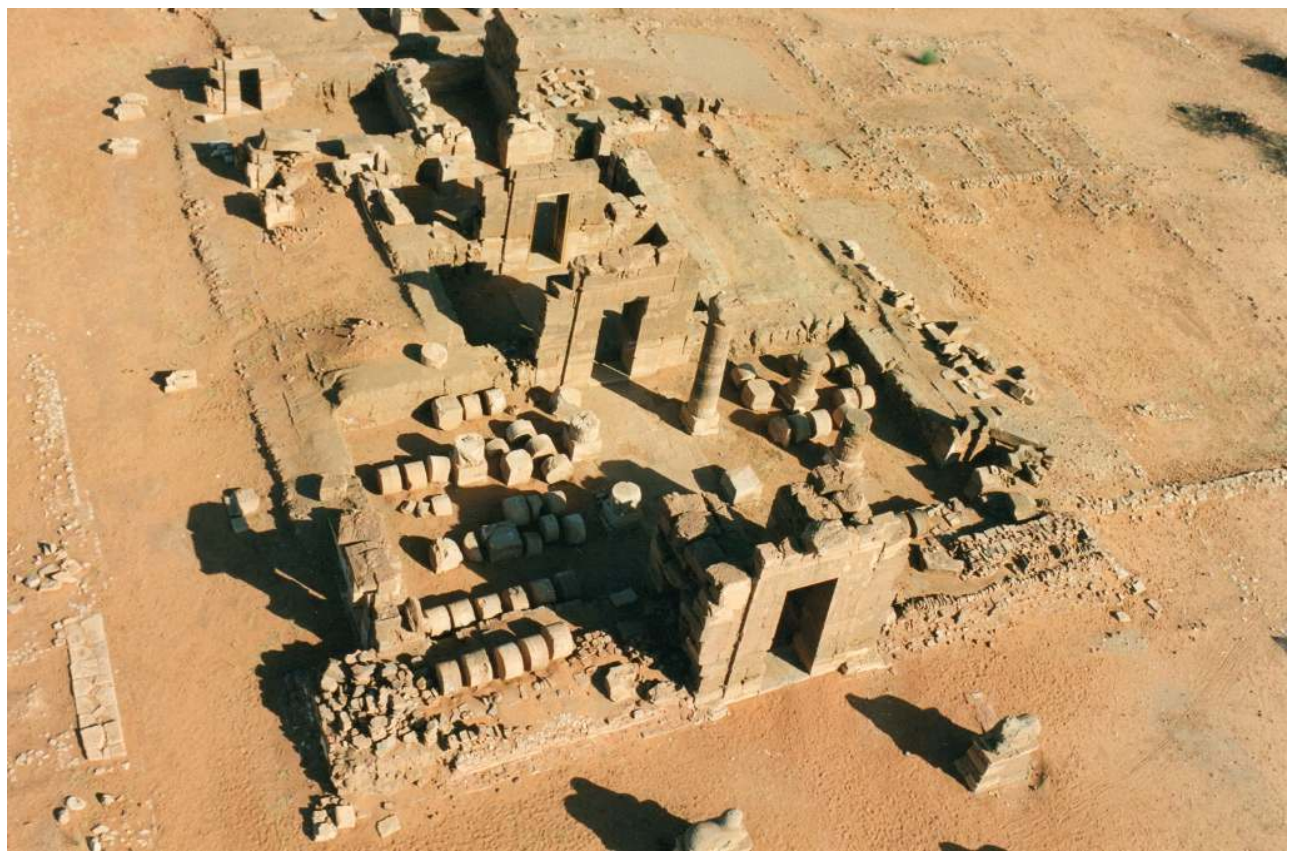

NAgA, TEMPLE D'AMON

(C) NAgA-PROJECT ; STAATLISCHES MUSEUM ÄgYPTISCHER KUNST, MÜNCHEN enfoui sous des pierres de plusieurs tonnes provenant du toit du sanctuaire ${ }^{38}$. Il est remplacé par une réplique sur le site, l'original se trouve au Musée national de Khartoum avec d'autres trouvailles provenant de Naga.

Un contre-temple est accolé directement au mur arrière du temple où une treizième statue de bélier était accessible à tous ceux qui ne pouvaient pénétrer dans les 
entrailles secrètes du temple. Ce mur s'est affaissé vers l'arrière et gît maintenant, toutes ses couches de briques soigneusement alignées sur le sol ${ }^{39}$. Un complexe de bâtiments construit contre le mur sud du temple d'Amon servait probablement d'habitation aux prêtres.

À quelques mètres du temple d'Amon, des déblais étaient visibles à la surface du sol au début des travaux de fouilles. Ce qui commença comme un nettoyage rapide et un enregistrement sur le plan s'est rapidement transformé en une opération de dégagement d'un temple additionnel de 1600 blocs de reliefs. Le programme décoratif de ce temple, nommé Naga $200^{40}$, montre une affinité avec le Temple du Lion, dont les reliefs des murs intérieurs et des murs extérieurs sont les plus beaux et les plus vastes exemples de l'art méroïtique en deux dimensions.

\section{Le Temple du Lion}

17 «Le premier objet qui attira mes regards, ce fut un temple tout couvert de sculptures égyptiennes, avec son pylône et un portique d'architecture gréco-romaine mêlée d'ornemens égyptiens ${ }^{41}$. » C'est ainsi que Cailliaud relate en 1822 son premier contact avec un temple quasiment intact, le mieux préservé de Naga et peut-être même du Royaume de Méroé. Encore aujourd'hui, sa publication, complétée des plans et des dessins effectués par Linant de Bellefonds quelques années auparavant ${ }^{42}$, reste une documentation historique importante du Temple du Lion (Naga 300) ${ }^{43}$. Parmi les quelques visiteurs suivants, le peintre Charles Gleyre effectue en 1835 des croquis du pylône et de la paroi ouest du temple. Le prince Hermann Ludwig Heinrich von PücklerMuskau donne également un rapport sur les reliefs du Temple du Lion et Richard Lepsius, lors de son expédition, en dresse un plan détaillé et en publie les reliefs.

Peu de voyageurs s'aventurent jusqu'à Naga, célèbre pour ses dangereux félins, et peutêtre est-ce pour cette raison que le Temple du Lion, dédié au Dieu Apedemak, est situé ici.

18 Malgré l'important travail de publication mené par le Tubinger Atlas des Vorderen Orients $^{44}$ sous la direction de I. Gamer Wallert et K. Zibelius, les fouilles du site ne commencent pas avant 1995.

Dès le début des travaux à Naga en 1995, il apparaît impératif avant d'entreprendre les fouilles, de documenter et de préserver ce qui était présent et visible, c'est-à-dire les quatre temples décrits par Cailliaud ${ }^{45}$. Ces quatre édifices représentent à eux seuls l'intégralité de l'art et de l'architecture méroïtique connus. Le Temple d'Amon a ensuite été fouillé parallèlement au Temple du Lion. Il s'agissait de nettoyer d'une part la zone autour du Temple du Lion afin de déterminer l'existence éventuelle d'éléments connectant les deux bâtiments, et d'autre part l'intérieur du Temple du Lion, ce qui a permis de mettre à jour 62 blocs.

19 Les fouilles ont également permis de mettre en évidence la présence d'un portique placé devant le pylône d'entrée du Temple du Lion. Ce portique était raccordé aux côtés étroits des deux tours du pylône, directement au-dessous des reliefs de fleurs d'acanthe desquelles s'élève une représentation du dieu Apédémak léocéphale à corps de serpent ${ }^{46}$. Le plan du portique atteste l'existence de saillies et de niches. Une rampe construite à l'intérieur, constituée de dalles de grès carrées et rectangulaires mène ensuite vers l'ouest, vers la porte du pylône et l'intérieur du temple qui comportait une salle unique. Au cours des fouilles ${ }^{47}$, quatre bases en grès ont été découvertes; elles 
étaient destinées à accueillir quatre colonnes en bois qui supportaient à l'origine un toit également en bois. Derrière les bases de colonnes à l'ouest, accolé au mur ouest au fond du temple, se trouvait un naos, probablement en bois, dont la fondation carrée en pierre $^{48}(2,60$ x 1,95 m) est le seul vestige (fig. 4). Deux stèles votives et deux bassins d'offrandes (dont l'un trouvé in situ dans le naos), attestent clairement l'existence du culte; deux plaques en faïence qui décoraient probablement la base du naos ont également été découvertes.

Fig. 4

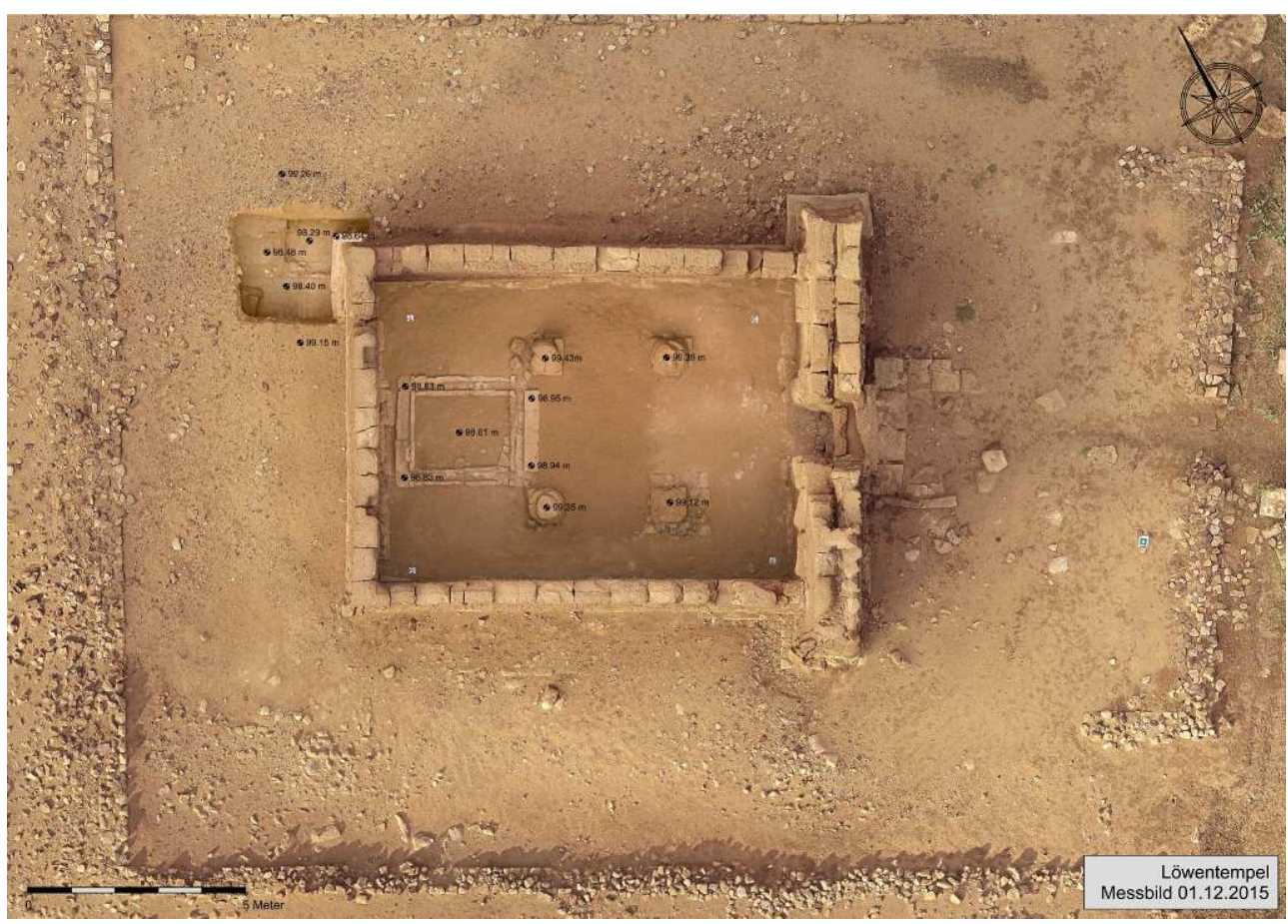

Naga, Temple du Lion, vue aérienne

(C) NAgA-PROJECT ; STAATLISCHES MUSEUM ÄgYPTISCHER KUNST, MÜNCHEN

20 Ce type de temple à salle unique, sans parallèle dans l'architecture égyptienne, est caractéristique des bâtiments dédiés au dieu purement méroïtique qu'est Apédémak ${ }^{49}$. Un mur d'enceinte rectangulaire a été découvert autour du Temple du Lion et de la chapelle, qui par la suite s'avéra de date moderne (1914). Une fois démoli, on a pu constater qu'au sud-ouest il avait été construit sur un mur ancien qui appartient au complexe du Temple du Lion.

Le Temple du Lion date de l'époque de Natakamani et de la Candace Amanitore (fig. 5). Tous les deux sont visibles sur la façade du pylône, le roi sur la tour sud et la reine sur la tour nord. À l'opposé des canons égyptiens, la reine est représentée de la même taille que le roi, avec une large carrure. Elle effectue le geste de "terrasser les ennemis ${ }^{50}$ ", symbolisant le pouvoir et traditionnellement associé au roi. Cette combinaison d'éléments d'origine égyptienne avec des formes méroïtiques n'est pas visible seulement dans la corpulence de la Candace, mais dans toute l'iconographie des figures $\mathrm{du}$ roi et des dieux. Ainsi, leurs costumes et leurs bijoux, ainsi que leurs couronnes et les enseignes ont des formes particulières et apparaissent à côté d'éléments égyptiens, ptolémaïques et romains. 
Fig. 5

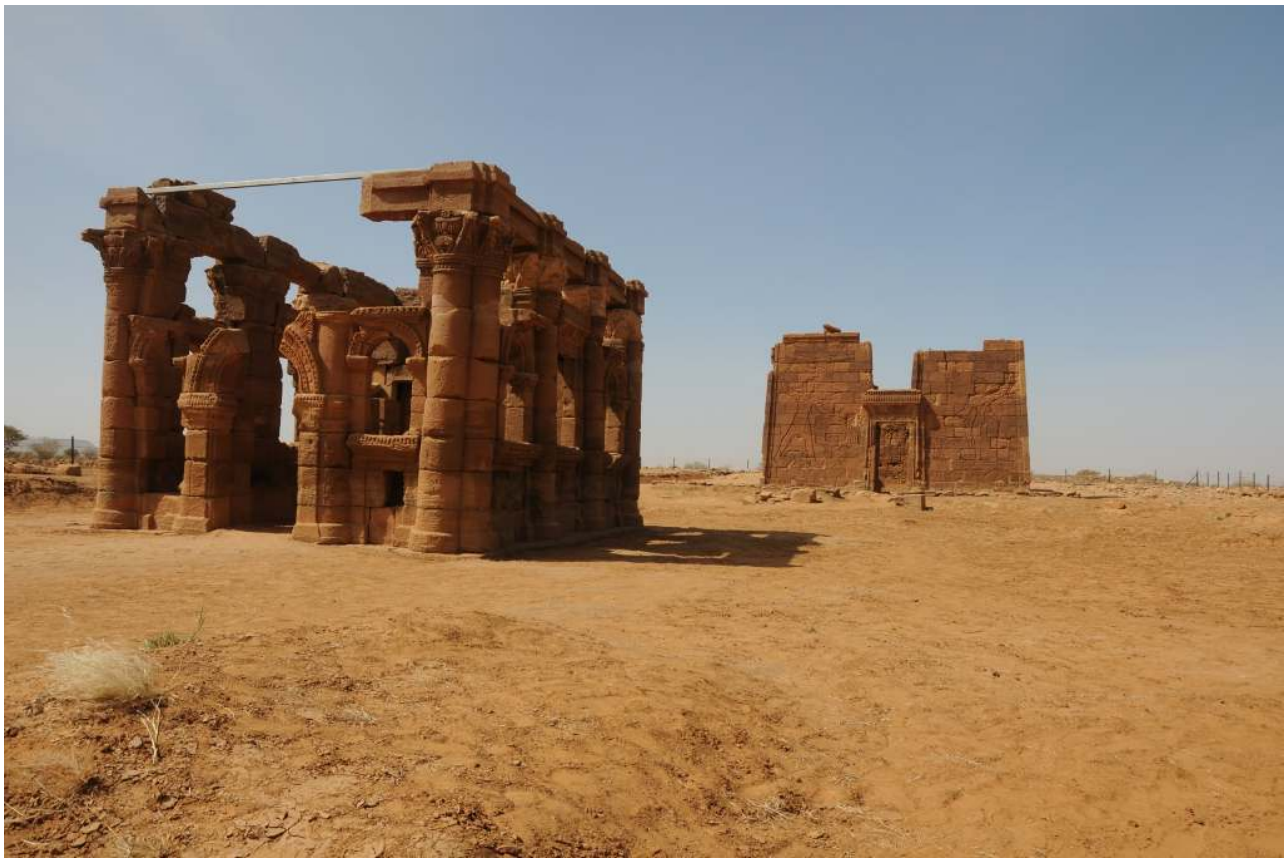

Naga, chapelle d'Hathor et Temple du Lion

(c) NAgA-PROJECT ; STAATLISCHES MUSEUM ÄgYPTISCHER KUNST, MÜNCHEN

De même, les deux représentations du dieu Apédémak sur les côtés du pylône, mentionnées plus haut, montrent elles aussi comment la décoration rompt avec les conventions égyptiennes, selon lesquelles le dieu devait être représenté sur le devant du pylône.

Les deux murs extérieurs longs, travaillés en relief en creux comme tous les murs extérieurs, montrent un groupe de dieux en pied devant la famille royale composée de Natakamani, suivie d'Amanitore et du prince Arikankharer. Comme dans le Temple d'Amon, et indiqué sur les tours du pylône du Temple du Lion, la division symétrique du temple en une moitié masculine sud et une moitié féminine nord se poursuit dans le reste de l'édifice. Ainsi, sur le mur sud, on voit d'est en ouest : Apédémak, Horus, Amon, Aqédis et Amon de Pnoubs devant la famille royale, et sur le mur nord les déesses Isis, Mout, Amésémi, Hathor et Satet ${ }^{51}$.

La scène la plus extraordinaire se trouve au revers du temple, centrée sur l'axe du bâtiment. Au centre se trouve le dieu-lion Apédémak, dépeint avec trois têtes et quatre bras afin de pouvoir ainsi se tourner du côté nord vers la Candace et le prince, du côté sud vers le roi et le prince ${ }^{52}$. La troisième tête regarde l'observateur d'en face, vers l'ouest. On peut postuler une quatrième face qui, elle, regardait à l'intérieur du temple, c'est-à-dire dans le sanctuaire.

Les reliefs à l'intérieur du temple, travaillés en haut-relief, n'ont été documentés que lors de l'expédition prussienne. Ils avaient déjà beaucoup souffert du temps de Bellefonds et de Cailliaud, plus que les reliefs en creux de l'extérieur. Cailliaud note : « En dedans les sculptures qui étaient toutes en relief, sont entièrement gâtées ${ }^{53}$ ». Les reliefs intérieurs n'intéressent tout d'abord pas Griffith lorsqu'il vient à Naga en 1910, puisqu'ils ne portent presque pas d'inscriptions. Ces reliefs, bien documentés par l'Université de Tübingen, permettent de noter une particularité intéressante, qui une fois de plus démontre le mélange d'éléments culturels divers: la représentation de 
dieux de face, avec les cheveux bouclés et la barbe pleine, sans doute une association avec le dieu romain Jupiter ou le dieu gréco-romain Sérapis ${ }^{54}$.

\section{Travaux actuels au Temple du Lion}

Pendant la campagne de 2015, l'intérieur du temple, ensablé lors de la campagne précédente pour le protéger, a été déblayé de nouveau afin de documenter l'état de la rangée inférieure de blocs de grès. On en a profité pour faire un sondage nord-sud entre les bases des colonnes afin d'étudier les fondations ${ }^{55}$. À une profondeur de $0,75 \mathrm{~m}$, une couche rougeâtre, compacte et plane a été mise à jour, un sondage fait en 1996 à l'intérieur du coin sud-ouest avait déjà fait apparaître des éléments semblables. Un troisième sondage, à l'extérieur du coin sud-ouest cette fois, a apporté le même résultat. La découverte de blocs décorés empêcha de creuser plus profondément, mais c'était assez pour confirmer que ce temple, à la différence du Temple du Lion 1200, avait été bâti sur un podium. Ces blocs appartiennent au complexe de bâtiments situés au sud du Temple du Lion qui comprend des bâtiments communs ou des maisons de prêtres dont l'entrée, orientée vers le temple, a pu être identifiée ainsi qu'un bloc provenant d'un linteau de porte orné d'uraeus.

Lors de la campagne suivante, un sondage a été creusé dans le coin opposé du temple, c'est-à-dire le coin nord-ouest. Cette fois, il a été possible d'aller plus en profondeur après avoir atteint la couche rouge compacte (à la profondeur attendue de $0,75 \mathrm{~m}$ ). Directement en-dessous de cette couche, on a mis au jour des lits réguliers de briques crues et cuites passant sous le Temple du Lion à un angle qui n'est pas parallèle à celui du mur nord. Les prochaines campagnes auront pour but de dégager le mur sur une plus grande longueur et d'éclaircir sa nature. Il s'agira aussi d'examiner les bâtiments au sud du Temple du Lion mentionnés ci-dessus. Il est en tout cas clair que cette nouvelle trouvaille - qu'il s'agisse d'un temple précurseur du Temple du Lion ou d'un autre monument - recule la date de fondation de Naga par rapport à ce que l'on supposait jusqu'ici. Ceci est avéré non seulement par la mention de Tolkte (Naga) à Moussaouarat es Soufra, mais aussi par des échantillons pris sous des bases de colonnes figuratives du bâtiment 4500 (pas encore examiné archéologiquement) qui ont été datés au carbone 14 .

La Chapelle d'Hathor, déjà mentionnée plusieurs fois (appelée par le passé «kiosque romain »), a été érigée à la même époque que le Temple du Lion. Elle incorpore de façon absolument unique des chapiteaux de type corinthien à des arcs en plein cintre romains et des éléments architecturaux égyptiens - un parfait exemple de pot-pourri architectural méroïtique typique de Naga. Les deux entrées de la chapelle sont diamétralement différentes: le portique ouest a une corniche à trois étages ornée d'uraeus et d'un disque solaire ailé, tous éléments typiques de l'architecture égyptienne, tandis que le portique est, avec son arc en plein cintre, ses pilastres à chapiteaux ioniens et éléments floraux, ressemble au portique d'une des premières basiliques.

Entre le Temple du Lion et la Chapelle d'Hathor se trouve un lieu d'offrande $(\text { Naga } 360)^{56}$. Orienté vers l'ouest, il est entouré sur trois côtés de blocs de grès de $0,35 \mathrm{~m}$ de haut recouverts d'enduit de calcaire blanc. On y a trouvé un grand nombre d'objets votifs déposés là par la population, dont des objets préhistoriques apparemment ramassés en tant que curiosités ${ }^{57}$. 


\section{Travaux actuels et objectifs futurs}

\section{NOTES}

1. Une stèle de Nastasen (musée Égyptien de Berlin, ÄM 2268) donne un nom de ville qui doit probablement être identifié avec Naga. Un texte du centre voisin de Musawwarat datant de 230 av. J.-C. nomme le dieu-lion "Apedemak de Tolkte", l'attribuant ainsi à Naga, qui est documenté dans d'autres textes plus tardifs sous son nom méroïtique de Tolkte. À Naga m ême, ce nom apparaît par exemple dans les inscriptions du Temple du Lion et du Temple d'Amon. 2. Sur l'histoire de la découverte et de la recherche scientifique sur Naga : Ingrid Gamer-Wallert, Karola Zibelius, Der Löwentempel von Naq'a in der Butana, Sudan I, Forschungsgeschichte und Topographie, supplément au Tübinger Atlas des Vorderen Orients, Série B (sciences humaines) 48 (1), Wiesbaden, Reichert, 1983.

3. Apôtres 8, 27.

4. Poncet voyagea de 1698 à 1700, accompagné du jésuite Charles Francis Xavérius de Brévedent, de l'Égypte jusqu'à la région de la capitale actuelle de Khartoum.

5. Theodor Krump, Hoher und Fruchtbahrer Palm-Baum, Augsburg, Joseph Gruber, 1710. Voir aussi Horst Lechner, «Ein von der Wissenschaft spät entdeckter Reisebericht. Die abenteuerliche 
Mission des Aichacher Franziskanerpaters Theodor Krump (1672-1724) nach Afrika ", dans Altbayern in Schwaben, Jahrbuch für Geschichte und Kultur 2007, Landratsamt Aichach-Friedberg, 2007, pp 47-62.

6. James Bruce, Travels to Discover the Source of the Nile, In the Years 1768, 1769, 1770, 1771, 1772 and 1773, vol. 5., Édimbourg, J. Ruthven, 1790.

7. Louis Maurice Adolphe Linant de Bellefonds, Journal d'un voyage à Méroé dans les années 1821 et 1822, M. Shinnie (dir.), Sudan Antiquities Service Occasional Papers 4, Khartoum, Sudan Antiquities Service, 1958. Pour les dessins de Naga faits par Linant de Bellefonds: http:// www.purl.org/yoolib/inha/7018, ils sont conservés au département des Antiquités égyptiennes du musée du Louvre. Les auteurs souhaitent remercier Marie Millet et Aminata Sackho-Autissier pour leur avoir signalé la présence de ce fonds dans les collections.

8. Frédéric Cailliaud, Voyage à Méroé au fleuve blanc, au dela de Fâzol dans le midi du Royaume de Sennâr, à Syouah et dans cinq autres oasis ; fait dans les années 1819, 1820, 1821 et 1822, Paris, Imprimerie royale, 1826-1927.

9. Ils se trouvent aujourd'hui dans la collection du château d'Alnwick.

10. Il fouille d'abord à Ouad ben Naga, Naga et Mousaouarat, puis à Méroé. Dans la nécropole du nord, il découvre le trésor d'Amanichakhéto (aujourd'hui dans les musées égyptiens de Berlin et de Munich) après avoir fait entièrement démanteler la pyramide de la reine (Pyramide N6).

11. Publiés dans: I. Gamer-Wallert, K. Zibelius, «Der Löwentempel von Naq'a in der Butana », op. cit. note 2.

12. Hermann von Pückler-Muskau, Aus Mehemed Alis Reich, vol. 3, Stuttgart, Hallberger, 1844.

13. Entre autres, les dessinateurs Ernst et Max Weidenbach, le peintre Otto Georgi et l'architecte Georg Gustav Erbkam

14. Karl Richard Lepsius, Denkmaeler aus Aegypten und Aethiopien: nach den Zeichnungen der von seiner Majestät dem Koenige von Preussen Friedrich Wilhelm IV nach diesen Ländern gesendeten und in den Jahren 1842-1845 ausgeführten wissenschaftlichen Expedition, Abth. 1-6, vol. 12, Berlin, 1849-1859.

15. P. Trémaux, Voyage en Éthiopie, au Soudan Oriental et dans La Nigritie I, Égypte et Éthiopie, Paris, Hachette, 1862.

16. Ernest Alfred Wallis Budge, The Egyptian Sudan I, Londres, K. Paul, 1907.

17. The Oriental Institute, 1905-1907, Breasted Expeditions to Egypt and the Sudan: A Photographic Study, Chicago, The University of Chicago Press, 1975. Consultable à cette adresse: https:// oi.uchicago.edu/collections/photographic-archives/1905-1907-breasted-expeditions-egypt-andsudan.

18. Francis Llewellyn Griffith, Meroitic Inscriptions, t. 2, Londres, The Egypt Exploration Fund, 1912.

19. Fritz Hintze, A Preliminary Report on the Butana Expedition 1958 of the Institute for Egyptology, Humboldt University Berlin, Kush 7, 1959, pp.171-196; F. Hintze, Vorbericht über die ButanaExpedition 1958 des Instituts für Ägyptologie der Humboldt-Universität zu Berlin, Forschen und Wirken, Festschrift zur 150-Jahr-Feier der Humboldt-Universität zu Berlin III, Berlin, 1960, pp. 361-399.

20. Theodor Kraus, « Der Kiosk von Naga », Archäologischen Anzeiger, 1964, p. 834.

21. I. Gamer-Wallert, K. Zibelius, « Der Löwentempel von Naq'a in der Butana », op. cit. note 2.

22. Ce projet est financé par la Deutsche Forschungsgemeinschaft.

23. Les fouilles sont financées par le Qatar Sudan Archaeological Project : http://www.qsap.qa/ en/.

24. Une grande exposition à Munich et à Berlin présenta les résultats des quinze premières années : cat. d'exp., Karla Kröper, Sylvia Schoske, Dietrich Wildung (dir.), Königsstadt Naga - NagaRoyal City. Grabungen in der Wüste des Sudan-Excavations in the Desert of the Sudan, Munich 15. avril - 31. juillet 2011, Berlin 31. août - 18. décembre 2011, Munich, Staatliches Museum Ägyptischer Kunst - Berlin, Kunstforum der Berliner Volksbank, 2011. Vous trouverez aussi un aperçu des résultats dans: K. Kroeper, «Rediscovery of the Kushite site - Naga, 15 years of 
excavation (1995-2010). Surprises and Innovations", dans Sudan \& Nubia 15, 2011. Des monographies sur les recherches sont en préparation et seront publiées par Ugarit-Verlag. 25. Voir dernièrement : D. Wildung, "Naga - Königsstadt in der Steppe ", dans Steffen Wenig, Karola Zibelius-Chen (dir.), Die Kulturen Nubiens - ein afrikanisches Vermächtnis, Dettelbach, Röll, 2013, pp. 415-428.

26. Alexandra Riedel, Jan Hamann, «From the quarry to the finished building. The ancient Meroitic stone masonry at the site of Naga/Sudan », dans Proceedings of the Third International Congress on Construction History, Cottbus, Brandenburg University of Technology, 2009, p. 200.

27. James Knudstad, Rosa Frey, «Naga Project (Sudan) - Egyptian Museum Berlin. The City Survey 1995-1996, Seasons 1 and 2 ", dans Archéologie du Nil Moyen 8, 1998, pp. 193-202.

28. À propos des hafirs, voir : Marion Hinkel, The archaeological map of the Sudan. Supplement II: Die Hafire im Sudan. Eine Untersuchung zur Geschichte des frühen Wasserspeicherbaus, Walter F. Reineke (dir.), 2 vol., Bonn, Habelt-Verlag, 2015.

29. K. Kröper, Lech Krzyzaniak, « Naga Project (Sudan) - Egyptian Museum Berlin. The Amun Temple Complex. Preliminary Report, Seasons 1 and 2 », dans Archéologie du Nil Moyen 8, 1998, p. 206 sqq.

30. K. Kröper, S. Schoske, D. Wildung, Königsstadt Naga, op. cit. note 24, fig. 46-48, cat. 18.

31. Idem, Ibidem, fig. 23-33, cat. 2-10.

32. D. Wildung, « Naga, die Stadt in der Steppe. Grabungen des Ägyptischen Museums im Sudan, Vorbericht II. Statuen aus dem Amun-Tempel », dans Jahrbuch der Berliner Museen 2001, NF 43, Berlin, Gebr. Mann Verlag, 2003, pp. 303-330.

33. Une stèle montre Amanchakhéto devant la déesse Amésémi, la deuxième la reine avec Amésémi devant le dieu Apedemak à tête de lion (exposé actuellement en tant que prêt permanent au musée Égyptien de Munich); voir : K. Kröper, S. Schoske, D. Wildung, Königsstadt Naga, op. cit. note 24, fig. 34 , cat. 11 et fig. 37, cat. 14 .

34. Idem, Ibidem, fig. 182, 183, cat. 109 ; exposée actuellement au musée Égyptien de Munich en tant que prêt permanent.

35. K. Kroeper, "Metamorphoses of the Amun Temple in Naga ", dans Isabelle Caneva, Alessandro Roccati (dir.), Acta Nubica - Proceedings of the X. International Conference of Nubian Studies, Rome 9-14 Sept. 2002, Rome, Libreria Dello Stato, 2006, pp. 287-295, fig. 16-19.

36. K. Kroeper, «The End of the Amun-Temple and beginning of Temple $200-\mathrm{C} 14$ dates from Naga », dans Cripel 26, 2007, pp. 231-242.

37. K. Kroeper, "L'"autel solaire" du Temple d'Amun à Naga ", dans Michel Baud (dir.), Méroé un empire sur le Nil, Milan, Officina Libraria, Paris, Musée du Louvre, 2010, p. 234 ; K. Kroeper, Rediscovery, op. cit. note 24, 95 f. ; K. Kröper, S. Schoske, D. Wildung, Königsstadt Naga, op.cit. note 24 , fig. 38.

38. D. Wildung, «Staatstragende Zweisamkeit. Der Altar im Sanktuar des Amuntempels in Naga, Sudan », dans Günther Schauerte, Moritz Wullen, Denken in Bildern. 31 Positionen zu Kunst, Museum und Wissenschaft, Ostfildern, Hatje Cantz Verlag, 2008, pp.242-249; K. Kröper, S. Schoske, D. Wildung, Königsstadt Naga, op. cit. note 24, fig. 97.

39. K. Kroeper, «Amun-Tempel Complex in Naga. Preliminary Report - Seasons 5-7 (1999-2001) », dans Archéologie du Nil Moyen 10, 2006, pp. 161-170.

40. Josefine Kuckertz, "Wiedergeburt eines Tempels. Rebirth of a Temple », dans K. Kröper, S. Schoske, D. Wildung, Königsstadt Naga, op. cit. note 24, pp. 66-85.

41. F. Cailliaud, Voyage à Méroé, op. cit. note 8, p. 122. Il atteint Naga le 22 mars 1822 après avoir entendu parler de l'existence du site par Louis-Maurice-Adolphe Linant de Bellefonds, qui a découvert la cité antique le 28 février 1822.

42. À consultez sur: INHA, Institut national d'histoire de l'art: http://bibliothequenumerique.inha.fr/collection/7018-dessins-executes-par-linant-de-bellefo/?n=14.

43. Selon la numérotation de Friedrich W. Hinkel. 
44. Griffith, Tubinger Atlas des Vorderen Orients, Wiesbaden, Reichert : que les murs extérieurs.

45. F. Cailliaud, Voyage à Méroé, op. cit. note 8 .

46. K. Kröper, S. Schoske, D. Wildung, Königsstadt Naga, op. cit. note 24, fig. 73, 74.

47. 1995 et 1996.

48. Friedrich W. Hinkel, « Naga Project (Sudan) - Egyptian Museum Berlin. The Lion Temple at Naga - Results of Investigations in 1996 », Archéologie du Nil Moyen 8, p. 223.

49. Par exemple, les temples du Gébel Hardan, Gébel Nasb es-Sami et Gébel Matruka.

50. K. Kröper, S. Schoske, D. Wildung, Königsstadt Naga, op. cit. note 24, fig. 151, 152.

51. Idem, Ibidem, fig. 150.

52. Idem, Ibidem, fig. 76.

53. F. Cailliaud, Voyage à Méroé, op. cit. note 8, t. III, p. 131.

54. K. Kröper, S. Schoske, D. Wildung, Königsstadt Naga, op. cit. note 24, fig. 160.

55. Le type de fondation ainsi que le manque de tambours en grès supposent une toiture en bois.

56. K. Kroeper, "Excavation of "Offering chapel 360" in Naga ", dans Julie Anderson, Derek Welsby (dir.), Fourth Cataract and Beyond. Proceedings of the 12th International Conference for Nubian Studies, Londres 2010, Londres, Peeters Publishers, 2014.

57. D. Wildung, "Prähistorische Votive in einem meroitischen Heiligtum », dans Daniel Polz, Stephan J. Seidlmayer (dir.), Gedenkschrift für Werner Kaiser, MDAIK 70/71 (2014/2015), 2016, pp. 463-469.

58. http://naga-project.com/de/das-museum/biennale-venedig-2016/.

\section{RÉSUMÉS}

En juin 2013, le projet Naga, qui se trouvait sous l'égide de l'Ägyptisches Museum und Papyrussammlung (musée Égyptien) de Berlin, est repris par le Staatliches Museum Ägyptischer Kunst (musée Égyptien de Munich). Le Professeur Dietrich Wildung (directeur du projet) et le Docteur Karla Kröper (direction des fouilles) dirigent les travaux au Soudan. Cet article veut donner un aperçu de l'histoire de la découverte de Naga, du site lui-même, des travaux des dernières années, des fouilles actuelles et des objectifs futurs du projet. Le site est inscrit au patrimoine mondial de l'Unesco. Le site du projet (www.naga-project.com) contient les actualités sur les travaux. Une liste de publications peut être consultée sur http://naga-project.com/nagaonline/publikationsliste/.

In June 2013, the Naga Project, which was under the aegis of the Ägyptisches Museum und Papyrussammlung in Berlin, was taken over by the Staatliches Museum Ägyptischer Kunst (in Munich. Professor Dietrich Wildung (scientific director) and Doctor Karla Kröper (field director) are directing the excavations in Sudan. This article aims to provide a brief overview of the discovery of Naga: the site itself; the excavations of the last few years, the current digs and the future objectives of the project. The website www.naga-project.com contains news about the project. A list of publications may be consulted on http://naga-project.com/naga-online/ publikationsliste/. 


\section{INDEX}

Mots-clés : Naga, Temple d'Amon, Temple des Lions, Chapelle d'Hathor, Apédémak, Natakamani, Candace Amanitore, Soudan, Méroé

Keywords : Naga, Temple of Amon, Temple of the Lions, Chapel of Hathor, Apedemak, Natakamani, Candace Amanitore, Sudan, Meroe

\section{AUTEURS}

\section{CHRISTIAN PERZLMEIER}

Christian Perzlmeier a étudié l'égyptologie à la Ludwig-Maximilians-Universität de Munich, les épigraphies égyptienne et copte à l'université de Munich. Il a travaillé dès 2002 en Égypte pour le DAIK à Dachour sous la direction de Rainer Stadelmann puis pour le projet du temple funéraire d'Amenhotep III sous la direction d'Hourig Sourouzian. Il est membre du Projet Naga et travaille au Staatliches Museum Ägyptischer Kunst de Munich depuis 2015.

Christian Perzlmeier studied Egyptology and Egyptian and Coptic epigraphy at LudwigMaximilians-Universität in Munich. In 2002 he began working in Dahshur, Egypt, for the Deutsche Archäologische Institut Kairo (DAIK) under the supervision of Rainer Stadelmann, then on the funerary temple of Amenhotep III project under the supervision of Hourig Sourouzian. He has been a member of Naga Project since 2015.

\section{ARNULF SCHLÜTER}

Arnulf Schlüter est reçu docteur en égyptologie en 2008 après avoir également étudié l'archéologie gréco-romaine et préhistorique à la Ludwig-Maximilians-Universität de Munich. Il est directeur adjoint du Staatliches Museum Ägyptischer Kunst de Munich et membre du projet Naga depuis 2013.

Arnulf Schlüter received his PhD in Egyptology in 2008; he had previously studied Egyptology, Greco-Roman and prehistoric archaeology at the Ludwig-Maximilians-Universität in Munich. He is assistant director of the Staatliches Museum Ägyptischer Kunst in Munich and is a member of the Naga Project since 2013. 